\title{
An Iterative Method for Shape Optimal Design of Stokes-Brinkman Equations with Heat Transfer Model
}

\author{
Wenjing Yan $\mathbb{D}^{1},{ }^{1}$ Feifei Jing, ${ }^{2}$ Jiangyong Hou, ${ }^{3}$ Zhiming Gao, ${ }^{4}$ and Nannan Zheng ${ }^{1}$ \\ ${ }^{1}$ School of Mathematics and Statistics, Xi'an Jiaotong University, Xi'an 710049, China \\ ${ }^{2}$ School of Mathematics and Statistics, Xi'an Key Laboratory of Scientific Computation and Applied Statistics, \\ Northwestern Polytechnical University, Xi'an 710129, China \\ ${ }^{3}$ School of Mathematics, Northwest University, Xi'an 710127, China \\ ${ }^{4}$ Institute of Applied Physics and Computational Mathematics, Beijing 100088, China \\ Correspondence should be addressed to Wenjing Yan; wenjingyan@xjtu.edu.cn
}

Received 19 April 2020; Accepted 7 May 2020; Published 29 June 2020

Academic Editor: Yumin Cheng

Copyright (C) 2020 Wenjing Yan et al. This is an open access article distributed under the Creative Commons Attribution License, which permits unrestricted use, distribution, and reproduction in any medium, provided the original work is properly cited.

\begin{abstract}
This work is concerned with the shape optimal design of an obstacle immersed in the Stokes-Brinkman fluid, which is also coupled with a thermal model in the bounded domain. The shape optimal problem is formulated and analyzed based on the framework of the continuous adjoint method, with the advantage that the computing cost of the gradients and sensitivities is independent of the number of design variables. Then, the velocity method is utilized to describe the domain deformation, and the Eulerian derivative for the cost functional is established by applying the differentiability of a minimax problem based on the function space parametrization technique. Moreover, an iterative algorithm is proposed to optimize the boundary of the obstacle in order to reduce the total dissipation energy. Finally, numerical examples are presented to illustrate the feasibility and effectiveness of our method.
\end{abstract}

\section{Introduction}

The optimal shape design for the fluid flows has wide applications in engineering design and computational fluid mechanics. The industrial applications include the design for wings profiles, impeller blades, and high-speed trains. In this paper, we focus on identifying the optimal shape of an obstacle located in the viscous and incompressible fluid, which is governed by Stokes-Brinkman equations strongly coupled with a thermal model. Our purpose is to effectively find the optimal shapes that minimize certain cost functional which may represent a given objective related to the specific characteristic features of the fluids, subject to mechanical and geometrical constraints.

Different methods have been proposed to numerically solve the shape optimal problems, such as generic algorithm [1], complex Taylor series expansion approach [2], automatic differentiation method [3], one-shot method [4, 5], level set method $[6,7]$, domain derivative method [8], and adjoint method [9-11]. Among the popularly used approaches, the adjoint method has received plenty of attention. Especially for the shape optimal control in fluids, the cost of computing the gradients and sensitivities is independent of the number of design variables. Jameson first applied this method to solve the shape design of aircraft [12]. Srinath and Mittal presented a numerical method for shape optimization for unsteady viscous flows which is based on the continuous adjoint approach [13]. Yagi and Kawahara utilized the adjoint method to identify the optimal shape for a body located in incompressible flow [14].

However, many authors considered the shape optimal problems in fluids without the heat transfer, steady state or not $[15,16]$. In Reference [17], Chenais et al. solved the shape optimal problem in a potential flow coupled with a thermal model. Moreover, the number of publications on shape optimal problems for Stokes-Brinkman equations is relatively small when compared to Stokes equations [18-20]. 
In shape optimization, the efficient computation requires a shape calculus which differs from its analog in vector spaces. The traditional approaches always involve the computation of the state derivative with respect to the domain, but the state parameters belong to the function spaces depending on the variable domain. Besides, the state differentiability is not necessary in many cases even if the state system is not differentiated. To avoid the differentiation of the state system, the adjoint method is employed to solve shape optimal problem, which just requires to solve only one extra adjoint system.

This paper is organized as follows. In Section 2, we briefly introduce the general approach of the shape optimal control problem in fluids. Section 3 briefly describes the shape optimal problem for Stokes-Brinkman equations and heat exchanges are considered. Section 4 is devoted to the velocity method which is used to perform the domain deformation. In addition, the definitions of Eulerian derivative and shape gradient are introduced. In Section 5, based on the minimax principle, the shape optimal problems can be expressed as the saddle point problems of some suitable Lagrangian functionals. Then, applying the function space parametrization technique and minimax differential theorem, we deduce the expression of shape gradient for the Lagrangian functional, which plays the key role of design variables in the optimal design framework. Finally, some numerical examples are presented to verify the effectiveness of the proposed method in Section 6.

\section{Shape Optimal Control Problem in Fluids}

In this section, we present the general structure to solve the optimal control problems, which will be applied to the particular case of shape optimal problem in Stokes-Brinkman flow with heat transfer in the following section.

Our work is to minimize a cost functional $\mathscr{J}$ which consists of the solution of the state equations:

$$
\left\{\begin{array}{l}
\min \mathscr{J}=\mathscr{J}(w, \varphi), \\
\mathscr{A} w=f+\mathscr{B} \varphi,
\end{array}\right.
$$

where $w$ is the state variable, $\mathscr{A}$ represents an elliptic differential operator, $f$ stands for the source term, and $\mathscr{B}$ denotes a differential operator acting on the control variable $\varphi$. Now, we introduce the Lagrangian functional $\mathscr{L}$ and Lagrangian multiplier $\lambda$ :

$$
\mathscr{L}(w, \lambda, \varphi):=\mathscr{J}(w, \varphi)+\langle\lambda, f+\mathscr{B} \varphi-\mathscr{A} w\rangle .
$$

For the linear case, problem (1) satisfies $\nabla \mathscr{L}(w, \lambda, \varphi)=0$. Suppose that $W$ and $V$ are two suitable Hilbert spaces; for $w \in W$ and $\varphi \in V$, we obtain the variational form for state equation (1):

$$
a(w, \psi)=(f, \psi)+b(\varphi, \psi), \quad \forall \psi \in V,
$$

where $(\cdot, \cdot)$ denotes the inner product, $a(\cdot, \cdot)$ is a bilinear form with respect to a linear elliptic operator, and $b(\varphi, \psi)=\langle\mathscr{B} \varphi, \psi\rangle$. Therefore,

$$
\mathscr{L}(w, \lambda, \varphi)=\mathscr{J}(w, \varphi)+b(\varphi, \lambda)+(f, \lambda)-a(w, \varphi) .
$$

We need to solve following problem to obtain the optimal solution:

$$
\operatorname{seek}(w, \lambda, \varphi) \in W \times W \times V, \quad \text { such that } \nabla \mathscr{L}(w, \lambda, \varphi)=0 \text {. }
$$

Usually, we can apply an iterative method to solve the control problem by choosing an initial value for the variable $\varphi^{0}$. At each step, we compute the state equations and then evaluate the cost functional and solve the adjoint equations. When $\varphi^{j}$ is available, we give a suitable stopping criterion and derive the cost functional derivative $\mathscr{J}^{\prime}[21,22]$.

\section{Shape Optimal Problem for Stokes-Brinkman Equations with Heat Transfer}

In this section, we focus on the shape optimal problem of modeling flow through porous and partially porous media, which is described by Stokes-Brinkman equations with heat transfer. Suppose that $\Omega \subset \mathbb{R}^{N}(N=2$ or 3$)$ is a bounded Lipschitz domain which is filled with the incompressible viscous fluid of the kinematic viscosity $\nu$. The boundary $\partial \Omega$ of the domain $\Omega$ is smooth and consists of four parts. $\Gamma_{n}$ denotes the inflow boundary, $\Gamma_{w}$ is the boundary corresponding to the fluid wall, $\Gamma_{o}$ represents the outflow boundary, and $\Gamma_{s}$ is the boundary of the obstacle $S$ which is to be optimized.

The fluid is described by the Stokes-Brinkman equations strongly coupled with a thermal model; the unknowns are the fluid velocity $u=\left(u_{1}, \ldots, u_{N}\right)^{T}: \Omega \longrightarrow \mathbb{R}^{N}$, the pressure $p: \Omega \longrightarrow \mathbb{R}$, and the temperature $T: \Omega \longrightarrow \mathbb{R}:$

$$
\begin{array}{rlrl}
-\operatorname{div} \sigma(u, p)+M u & =\lambda j T & \text { in } \Omega, \\
\operatorname{div} u & =0 & & \text { in } \Omega, \\
u & =0 & & \text { on } \Gamma_{w} \cup \Gamma_{s}, \\
u & =g, & & \text { on } \Gamma_{n}, \\
-\alpha \Delta T+u \cdot \nabla T & =0 & & \text { in } \Omega, \\
\frac{\partial T}{\partial n} & =0 & & \text { on } \Gamma_{o} \cup \Gamma_{s}, \\
T & =T_{1} & & \text { on } \Gamma_{w}, \\
T & =T_{2} & & \text { on } \Gamma_{n},
\end{array}
$$

where the stress tensor $\sigma(u, p)$ is defined by $\sigma(u, p):=-$ $p I+2 v \in(u)$ with the rate of deformation tensor $\epsilon(u):=\left(D u+D^{T} u\right) / 2, D^{T} u$ denotes the transpose of the matrix $D u$, and $I$ is the identity tensor. The matrix-valued function $M: \Omega \longrightarrow \mathbb{R}^{N \times N}$, $\alpha$ denotes the inverse of Peclet number, $\lambda$ is the Grashof number, and $j$ equals $(0,1)^{T}$.

In this paper, our work is to identify the optimal shape of the boundary $\Gamma_{s}$ that minimizes the following cost functional $J$ : 


$$
\min _{\Omega \in \mathscr{O}} J(\Omega)=2 v \int_{\Omega}|\in(\vec{u})|^{2} \mathrm{~d} x+\frac{1}{2} \int_{\Omega}|\nabla T|^{2} \mathrm{~d} x
$$

where $\vec{u}$ and $T$ denote the velocity and the temperature and $\epsilon(u)$ is the rate of deformation tensor. The shape admissible set $\mathcal{O}$ is given by

$$
\mathcal{O}:=\left\{\Omega \subset \mathbb{R}^{N}: \Gamma_{n} \cup \Gamma_{w} \cup \Gamma_{o} \text { is fixed, } \int_{\Omega} \mathrm{d} x=\text { constant }\right\} .
$$

This type of optimal problem often occurs in the design and control of many industrial equipment. The weak formulation associated with (6)-(13) can be written as

$$
\left\{\begin{array}{l}
\text { Find }(u, p, T) \in V_{g}(\Omega) \times Q(\Omega) \times \in H^{1}(\Omega), \text { such that, } \\
\int_{\Omega}\left[2 v \in(u): \in(v)+v^{T} M u-p \operatorname{div} v\right] \mathrm{d} x=\int_{\Omega} \lambda T j \cdot v \mathrm{~d} x, \quad \forall v \in V_{0}(\Omega), \\
\int_{\Omega} \operatorname{div} u q \mathrm{~d} x=0, \quad \forall q \in Q(\Omega), \\
\int_{\Omega}(\alpha \nabla T \cdot \nabla S+u \cdot \nabla T S) \mathrm{d} x=0, \quad \forall S \in H^{1}(\Omega),
\end{array}\right.
$$

where the functional spaces are defined as follows:

$$
\begin{aligned}
V_{0}(\Omega): & =\left\{w \in\left(H^{1}(\Omega)\right)^{2}: w=0 \text { on } \Gamma_{w} \cup \Gamma_{s} \cup \Gamma_{n}\right\}, \\
Q(\Omega) & :=\left\{p \in L^{2}(\Omega): \int_{\Omega} p \mathrm{~d} x=0\right\}, \\
V_{g}(\Omega): & =\left\{w \in\left(H^{1}(\Omega)\right)^{2}: w=0 \text { on } \Gamma_{w} \cup \Gamma_{s}, w=\text { gon } \Gamma_{n}\right\} .
\end{aligned}
$$

Shape optimal problems usually involve very large computational costs, besides the numerical approximation of partial differential equations and optimization. To avoid the differentiation of the state system and save the computational cost, the adjoint method applied to solve shape optimal problem can be summarized as follows: first, we establish the saddle point problem and the Lagrangian functional associated with the cost functional and weak form of the state system. Then, we are able to perform the shape sensitivity analysis of the Lagrangian functional by the minimax principle concerning the differentiability problem. Last but not least, applying the function space technique, we obtain the Euler derivative of cost functional by the first variation of the cost functional with respect to the domain.

First, we give the following Lagrangian functional which is associated with (14) and (16):

$$
L(\Omega, u, p, T, v, q, S)=J(\Omega)-W(\Omega, u, p, T, v, q, S),
$$

where

$$
W(\Omega, u, p, T, v, q, S)=\int_{\Omega}\left[2 v \in(u): \in(v)+v^{T} M u-p \operatorname{div} v-\operatorname{div} u q-\lambda T j \cdot v\right] \mathrm{d} x+\int_{\Omega}(\alpha \nabla T \cdot \nabla S+u \cdot \nabla T S) \mathrm{d} x
$$

Now, problem (18) can be transformed into the saddle point form:

$$
\min _{\Omega \in \mathcal{O}} \min _{(u, p, T) \in V_{g}(\Omega) \times Q(\Omega) \times H^{1}(\Omega)} \max _{(v, q, S) \in V_{0}(\Omega) \times Q(\Omega) \times H^{1}(\Omega)} L(\Omega, u, p, T, v, q, S) .
$$

Then, we use the minimax framework to avoid the analysis of the state derivative with respect to the variable domains and establish the first optimality condition of the shape optimal problem to deduce the adjoint equations:

$$
\min _{(u, p, T) \in V_{g}(\Omega) \times Q(\Omega) \times H^{1}(\Omega)} \max _{(v, q, S) \in V_{0}(\Omega) \times Q(\Omega) \times H^{1}(\Omega)} L(\Omega, u, p, T, v, q, S) .
$$

Since the adjoint equations are defined from the Euler-Lagrange equations of the corresponding Lagrange functional $L$, the variation of $L$ with respect to $(v, q, S)$ can recover the state system and its weak formulation. 
Furthermore, we can differentiate $L$ with respect to the state variables $(u, p, T)$ to deduce the adjoint state system.

Differentiating Lagrangian functional $L$ with respect to $p$ in the direction $\delta p$, we have

$$
\frac{\partial L}{\partial p}(\Omega, u, p, T, v, q, S) \cdot \delta p=\int_{\Omega} \delta p \operatorname{div} v \mathrm{~d} x=0 .
$$

Owing to $\delta p$ with compact support in $\Omega$, it leads to $\operatorname{div} v=0$. Moreover, we differentiate $L$ with respect to $u$ in the direction $\delta u$ and apply Green formula:

$$
\begin{aligned}
& \frac{\partial L}{\partial u}(\Omega, u, p, T, v, q, S) \cdot \delta u \\
& =\int_{\Omega}(-2 v \Delta u+v \Delta v-\nabla q-M v) \cdot \delta u \mathrm{~d} x-\int_{\Omega} S \nabla T \cdot \delta u \mathrm{~d} x-2 v \int_{\partial \Omega} \in(v) \cdot n \cdot \delta u \mathrm{~d} s+4 v \int_{\partial \Omega} \in(u) \cdot n \cdot \delta u \mathrm{~d} s+\int_{\nabla \Omega} q \delta u \cdot n \mathrm{~d} s \\
& =\int_{\Omega}(-2 v \Delta u+v \Delta v-\nabla q-M v-S \nabla T) \cdot \delta u \mathrm{~d} x \\
& \quad-\int_{\partial \Omega} \sigma(v, q) \cdot n \cdot \delta u \mathrm{~d} s+4 v \int_{\partial \Omega} \in(u) \cdot n \cdot \delta u \mathrm{~d} s .
\end{aligned}
$$

$$
\sigma(v, q) \cdot n-4 v \in(u) \cdot n=0
$$

Since $\delta u$ has compact support in $\Omega$, we obtain

$$
-v \Delta v+M v+\nabla q+S \nabla T=-2 v \Delta u \text {. }
$$

To vary $\delta u$ on the boundary $\Gamma_{o}$, we deduce $T$ :

Similarly, we obtain the adjoint equation with respect to

$$
\begin{aligned}
& \frac{\partial L}{\partial T}(\Omega, u, p, T, v, q, S) \cdot \delta T \\
& =\int_{\Omega}(\alpha \Delta S+u \cdot \nabla S+\lambda T j \cdot v) \cdot \delta T \mathrm{~d} x-\int_{\Gamma_{o} \cup \Gamma_{s}}\left(\alpha \frac{\partial S}{\partial n}+u \cdot S \cdot n\right) \delta T \mathrm{~d} s \\
& \quad-\int_{\Omega} \Delta T \cdot \delta T \mathrm{~d} x+\int_{\partial \Omega} \nabla T \cdot \delta T \cdot n \mathrm{~d} s .
\end{aligned}
$$

Finally, we derive the following adjoint state system associated with (6)-(13):

$$
\begin{aligned}
& \begin{cases}-v \Delta v+M v+\nabla q+S \nabla T=-2 v \Delta u \\
\operatorname{div} v=0 & \text { in } \Omega, \\
\sigma(v, q) \cdot n-4 v \in(u) \cdot n=0 & \text { in } \Omega, \\
v=0 & \text { on } \Gamma_{o},\end{cases} \\
& \begin{cases}\alpha \Delta S-u \cdot \nabla S-\lambda j \cdot v=-\Delta T & \text { in } \Omega, \\
\alpha \frac{\partial S}{\partial n}+u \cdot S \cdot n=\nabla T \cdot n & \text { on } \Gamma_{o} \cup \Gamma_{w}, \\
S=0 & \text { on } \Gamma_{n} \cup \Gamma_{w} .\end{cases}
\end{aligned}
$$

\section{The Velocity Method}

In this section, we will apply the velocity method to describe the domain deformation. For shape optimal problem, the set of domain $\Omega$ is not a vectorial space, but we need an expression of the differential of the cost functional. In order to overcome this difficulty, we define the derivative of a realvalued function with respect to the domain so that we can present the differential expression for the cost functional to establish a gradient-type algorithm.

Let boundary $\nabla \Omega$ be piecewise $C^{k}$ and the velocity field $V \in E^{k}:=C\left([0, \tau] ;\left[\mathscr{D}^{k}(\bar{\Omega})\right]^{N}\right)$, where $\tau$ is a small positive real number and $\left[\mathscr{D}^{k}(\bar{\Omega})\right]^{N}$ denotes the space of all $k$-times continuous differentiable functions with compact support contained in $\Omega$. The velocity field

$$
V(\epsilon)(x)=V(\epsilon, x), \quad x \in \Omega, \epsilon \geq 0
$$


belongs to $\left[\mathscr{D}^{k}(\bar{\Omega})\right]^{N}$ for each $\epsilon$. It can generate transformations $F_{\epsilon}(V) X=x(\epsilon, X)$ through the following dynamical system:

$$
\frac{\mathrm{d} x}{\mathrm{~d} \epsilon}(\epsilon, X)=V(\epsilon, x(\epsilon)), \quad x(0, X)=X,
$$

with the initial value $X$. The flow with respect to $V$ can be defined as the mapping $F_{\epsilon}: \mathbb{R}^{N} \longrightarrow \mathbb{R}^{N}$ with $F_{\epsilon}(X)=x(\epsilon, X)$, where $x(\epsilon, X)$ is the solution of $(30)$. The transformed domain $F_{\epsilon}(V)(\Omega)$ can be denoted by $\Omega_{\epsilon}(V)$ at $\epsilon \geq 0$, and its boundary $\Gamma_{\epsilon}:=F_{\epsilon}(\nabla \Omega)$.

Next, we introduce two definitions for shape sensitivity analysis. The Eulerian de rivative of the cost functional $J(\Omega)$ at $\Omega$ for the velocity field $\vec{V}$ is defined as [23]

$$
\lim _{\epsilon \searrow 0} \frac{1}{\epsilon}\left[J\left(\Omega_{\epsilon}\right)-J(\Omega)\right]:=\mathrm{d} J(\Omega ; V) .
$$

Moreover, if the map $V \mapsto \mathrm{d} J(\Omega ; V): E^{k} \longrightarrow \mathbb{R}$ is linear and continuous, $J$ is shape differentiable at $\Omega$. In the distributional sense, it leads to

$$
\mathrm{d} J(\Omega ; V)=\langle\nabla J, V\rangle\left(\mathscr{D}^{k}(\bar{D})^{N}\right) \times \mathscr{D}^{k}(\bar{D})^{N} .
$$

When $J$ has a Eulerian derivative, $\nabla J$ is called the shape gradient of $J$ at $\Omega$.

\section{Function Space Parametrization}

In this section, we derive the expression of the shape gradient for the cost functional $J(\Omega)$ by the function space parametrization techniques.

The velocity method is applied to describe the domain deformations. We only perturb the boundary $\Gamma_{s}$ and consider the mapping $F_{\epsilon}(V)$ and the flow of the velocity field:

$$
V \in V_{\text {ad }}:=\left\{V \in C^{0}\left([0, \tau] ;\left[C^{2}\left(\mathbb{R}^{N}\right)\right]^{N}\right): V=0 \text { in the neighorhood of } \Gamma_{n} \cup \Gamma_{w} \cup \Gamma_{o}\right\} .
$$

The perturbed domain is denoted by $\Omega_{\epsilon}=F_{\epsilon}(V)(\Omega)$.
We aim to evaluate the derivative of $j(\epsilon)$ with respect to $\epsilon$, where

$$
j(\epsilon):=\min _{\left(u_{\epsilon}, p_{\epsilon}, T_{\epsilon}\right) \in V_{g}\left(\Omega_{\epsilon}\right) \times Q\left(\Omega_{\epsilon}\right) \times H^{1}\left(\Omega_{\epsilon}\right)} \max _{\left(v_{\epsilon}, q_{\epsilon}, S_{\epsilon}\right) \in V_{0}\left(\Omega_{\epsilon}\right) \times Q\left(\Omega_{\epsilon}\right) \times H^{1}\left(\Omega_{\epsilon}\right)} L\left(\Omega_{\epsilon}, u_{\epsilon}, p_{\epsilon}, T_{\epsilon}, v_{\epsilon}, q_{\epsilon}, S_{\epsilon}\right),
$$

and $\left(u_{\epsilon}, p_{\epsilon}, T_{\epsilon}\right)$ and $\left(v_{\epsilon}, q_{\epsilon}, S_{\epsilon}\right)$ satisfy corresponding state and adjoint systems on the perturbed domain $\Omega_{\epsilon}$, respectively. However, the Sobolev spaces $V_{g}\left(\Omega_{\epsilon}\right), V_{0}\left(\Omega_{\epsilon}\right)$, $\mathrm{Q}\left(\Omega_{\epsilon}\right)$, and $W\left(\Omega_{\epsilon}\right)$ depend on the perturbation parameter $\epsilon$. Consequently, we need to apply the function space parametrization technique to get rid of it. The advantage of this technique is being able to transport different quantities defined on the variable domain $\Omega_{\epsilon}$ back into the reference domain $\Omega$ which is entirely unrelated to $\epsilon$. Then, we can employ the differential calculus since the functionals involved are defined in a fixed domain $\Omega$ with respect to the parameter $\epsilon$.

Now, we define the following parametrization functions:

$$
\begin{aligned}
V_{g}\left(\Omega_{\epsilon}\right) & =\left\{u \circ F_{\epsilon}^{-1}: u \in V_{g}(\Omega)\right\}, \\
V_{0}\left(\Omega_{\epsilon}\right) & =\left\{v \circ{ }_{\epsilon}^{-1}: v \in V_{0}(\Omega)\right\}, \\
Q\left(\Omega_{\epsilon}\right) & =\left\{p \circ F_{\epsilon}^{-1}: p \in Q(\Omega)\right\}, \\
H^{1}\left(\Omega_{\epsilon}\right) & =\left\{T \circ F_{\epsilon}^{-1}: T \in H^{1}(\Omega)\right\},
\end{aligned}
$$

where "o" denotes the composition of the two maps.

Note that $F_{\epsilon}$ and $F_{\epsilon}^{-1}$ are diffeomorphisms, so the parametrization will not change the value of the saddle point. We can rewrite (34) as

$$
j(\epsilon)=\min _{(u, p, T)} \max _{(v, q, S)} L\left(\Omega_{\epsilon}, u \circ F_{\epsilon}^{-1}, p \circ F_{\epsilon}^{-1}, T \circ F_{\epsilon}^{-1}, v \circ F_{\epsilon}^{-1}, q \circ F_{\epsilon}^{-1}, S \circ F_{\epsilon}^{-1}\right),
$$

where the Lagrangian functional

$$
L\left(\Omega_{\epsilon}, u \circ F_{\epsilon}^{-1}, p \circ F_{\epsilon}^{-1}, T \circ F_{\epsilon}^{-1}, v \circ F_{\epsilon}^{-1}, q \circ F_{\epsilon}^{-1}, S \circ F_{\epsilon}^{-1}\right)=I_{1}(\epsilon)+I_{2}(\epsilon)+I_{3}(\epsilon)
$$

with 


$$
\begin{aligned}
& I_{1}(\epsilon):=2 v \int_{\Omega_{e}}\left|\epsilon\left(u \circ F_{\epsilon}^{-1}\right)\right|^{2} \mathrm{~d} x+\frac{1}{2} \int_{\Omega_{\epsilon}}\left|\nabla\left(T \circ F_{\epsilon}^{-1}\right)\right|^{2} \mathrm{~d} x, \\
& I_{2}(\epsilon):=-\int_{\Omega_{t}}\left[2 v \epsilon\left(v \circ F_{\epsilon}^{-1}\right): \epsilon\left(u \circ F_{\epsilon}^{-1}\right)+\left(v \circ F_{\epsilon}^{-1}\right)^{T} M\left(u \circ F_{\epsilon}^{-1}\right)-\left(p \circ F_{\epsilon}^{-1}\right) \operatorname{div}\left(v \circ F_{\epsilon}^{-1}\right)-\operatorname{div}\left(u \circ F_{\epsilon}^{-1}\right)\left(q \circ F_{\epsilon}^{-1}\right)-\lambda j\left(T \circ F_{\epsilon}^{-1}\right)\left(v \circ F_{\epsilon}^{-1}\right)\right] \mathrm{d} x \\
& I_{3}(\epsilon):=-\int_{\Omega_{e}}\left[\alpha \nabla\left(T \circ F_{\epsilon}^{-1}\right) \cdot \nabla\left(S \circ F_{\epsilon}^{-1}\right)+\left(u \circ F_{\epsilon}^{-1}\right) \cdot \nabla\left(T \circ F_{\epsilon}^{-1}\right)\left(S \circ F_{\epsilon}^{-1}\right)\right] \mathrm{d} x .
\end{aligned}
$$

Next work is to differentiate the perturbed Lagrangian functional $L\left(\Omega_{\epsilon}, u \circ F_{\epsilon}^{-1}, p \circ F_{\epsilon}^{-1}, T \circ F_{\epsilon}^{-1}, v \circ F_{\epsilon}^{-1}, q \circ F_{\epsilon}^{-1}\right.$, $S \circ F_{\epsilon}^{-1}$ ), so we introduce the following Hadamard formula to perform the differentiation:

$$
\frac{\mathrm{d}}{\mathrm{d} \epsilon} \int_{\Omega_{\epsilon}} \mathfrak{T}(\epsilon, x) \mathrm{d} x=\int_{\Omega_{\epsilon}} \frac{\nabla \mathfrak{T}}{\nabla \epsilon}(\epsilon, x) \mathrm{d} x+\int_{\nabla \Omega_{\epsilon}} \mathfrak{T}(\epsilon, x) \mathbf{V} \cdot n_{\epsilon} \mathrm{d} s
$$

for a sufficiently smooth functional $\mathfrak{I}:[0, \tau] \times \mathbb{R}^{N} \longrightarrow \mathbb{R}$. Applying (39), we have

$$
\left.\partial_{\epsilon} L\left(\Omega_{\epsilon}, u \circ F_{\epsilon}^{-1}, p \circ F_{\epsilon}^{-1}, T \circ F_{\epsilon}^{-1}, v \circ F_{\epsilon}^{-1}, q \circ{ }_{\epsilon}^{-1}, S \circ F_{\epsilon}^{-1}\right)\right|_{\epsilon=0}=I_{1}^{\prime}(0)+I_{2}^{\prime}(0)+I_{3}^{\prime}(0),
$$

where

$$
I_{1}^{\prime}(0)=4 v \int_{\Omega} \epsilon(u): \epsilon(-D u \cdot \mathbf{V}) \mathrm{d} x+2 v \int_{\Gamma_{s}}|\epsilon(\vec{u})|^{2} \mathbf{V}_{n} \mathrm{~d} s+\int_{\Omega} \nabla T \cdot \nabla(-D T \cdot \mathbf{V}) \mathrm{d} x+\frac{1}{2} \int_{\Gamma_{s}}|\nabla T|^{2} \mathbf{V}_{n} \mathrm{~d} s
$$

$I_{2}^{\prime}(0)=-\int_{\Omega}\left[2 v \in(-D u \cdot \mathbf{V}) \cdot \epsilon(v)+2 v \epsilon(u) \cdot \epsilon(-D v \cdot \mathbf{V})+v^{T} M \cdot(-D u \cdot \mathbf{V})+M u \cdot(-D v \cdot \mathbf{V})-(-\nabla p \cdot \mathbf{V}) \operatorname{div} v\right.$ $-p \operatorname{div}(-D v \cdot \mathbf{V})-(-\nabla q \cdot \mathbf{V}) \operatorname{div} u-\lambda j T \cdot(-D v \cdot \mathbf{V})-\lambda j(-D T \cdot \mathbf{V}) \cdot v-q \operatorname{div}(-D u \cdot \mathbf{V})] \mathrm{d} x$ $+\int_{\Gamma_{s}}\left(-2 v \in(u): \in(v)-v^{T} M u+p \operatorname{div} v+\operatorname{div} u q\right) \mathbf{V}_{n} \mathrm{~d} s$,

$$
\begin{aligned}
I_{3}^{\prime}(0)= & -\int_{\Omega} \alpha \nabla(-D S \cdot \mathbf{V}) \cdot \nabla T \mathrm{~d} x-\int_{\Omega} \alpha \nabla S \cdot \nabla(-D T \cdot \mathbf{V}) \mathrm{d} x-\int_{\Omega}(-D u \cdot \mathbf{V}) \cdot \nabla T S \mathrm{~d} x-\int_{\Omega} u \cdot \nabla(-D T \cdot \mathbf{V}) S \mathrm{~d} x+\int_{\Omega} u \\
& \cdot \nabla T(D S \cdot \mathbf{V}) S \mathrm{~d} x-\int_{\Gamma_{s}} u \cdot(-D T \cdot \mathbf{V}) S \cdot n \mathrm{~d} s-\int_{\Gamma_{s}}(\alpha \nabla T \cdot \nabla S+u \cdot \nabla T S) \mathbf{V}_{n} \mathrm{~d} s-\int_{\Gamma_{s}} \alpha \nabla T \cdot(-D S \cdot \mathbf{V}) \cdot n \mathrm{~d} x-\int_{\Gamma_{s}} \alpha \nabla S \\
& \cdot(-D T \cdot V) \cdot n \mathrm{~d} x .
\end{aligned}
$$

In order to simplify the above identities, we introduce the following lemma.

Lemma 1 (see [23]). If vector functions $u$ and $v$ vanish on the boundary $\Gamma_{s}$, the following identities hold on the boundary $\Gamma_{s}$ :

$$
\begin{array}{r}
D u \cdot \mathbf{V} \cdot n=\operatorname{div} u \mathbf{V}_{n} \\
\epsilon(u): \epsilon(v)=(\epsilon(u) \cdot n) \\
(\epsilon(u) \cdot n) \cdot(D v \cdot \mathbf{V})=(\epsilon(u) \cdot n) \\
\text { We apply Lemma } 1 \text { and obtain }
\end{array}
$$$$
\epsilon(u): \in(v)=(\epsilon(u) \cdot n) \cdot(\epsilon(v) \cdot n),
$$$$
(\epsilon(u) \cdot n) \cdot(D v \cdot \mathbf{V})=(\epsilon(u) \cdot n) \cdot(\epsilon(v) \cdot n) \mathbf{V}_{n} \text {. }
$$

$$
\begin{aligned}
I_{1}^{\prime}(0) & =-2 v \int_{\Omega} \Delta u \cdot(-D u \cdot \mathbf{V}) \mathrm{d} x-2 v \int_{\Gamma_{s}}|\epsilon(u)|^{2} \mathbf{V}_{n} \mathrm{~d} s-\int_{\Omega} \Delta T \cdot(-D T \cdot \mathbf{V}) \mathrm{d} x \\
& +\int_{\Gamma_{s}}(\nabla T \cdot n) \cdot(-\nabla T \cdot \mathbf{V}) \mathrm{d} s+\frac{1}{2} \int_{\Gamma_{s}}|\nabla T|^{2} \mathbf{V}_{n} \mathrm{~d} s .
\end{aligned}
$$


Recalling $(u, p, T)$ and $(v, q, S)$ satisfies the state and adjoint system, respectively, and (42) can be reduced to

$$
\begin{aligned}
I_{2}^{\prime}(0)= & \int_{\Omega}[(v \Delta u-M u-\nabla p-\lambda j T) \cdot(-D v \cdot \mathbf{V})] \mathrm{d} x-\int_{\Gamma_{s}}(2 v \in(u): \in(v)) \mathbf{V}_{n} \mathrm{~d} s \\
& +\int_{\Omega}\left[\left(v \Delta v-v^{T} M-\nabla q\right)\right] \cdot(-D u \cdot \mathbf{V}) \mathrm{d} x+\int_{\Omega} \lambda j(-D T \cdot \mathbf{V}) \cdot v \mathrm{~d} x-\int_{\Gamma_{s}}[\sigma(u, p) \cdot n \cdot(-D v \cdot \mathbf{V})+\sigma(v, q) \cdot n \cdot(-D u \cdot \mathbf{V}) \mathrm{d} s] \\
= & \int_{\Omega}(2 v \Delta u+\nabla T S)(-D u \cdot \mathbf{V}) \mathrm{d} x+\int_{\Omega} \lambda j(-D T \cdot \mathbf{V}) \cdot \vec{v} \mathrm{~d} x \\
& +\int_{\Gamma_{s}}(2 v \in(u): \in(v)) \mathbf{V}_{n} \mathrm{~d} s .
\end{aligned}
$$

Similarly, (43) can be rewritten as

$$
\begin{aligned}
I_{3}^{\prime}(0)= & \int_{\Omega}(\alpha \Delta T-u \cdot \nabla T)(-D S \cdot \mathbf{V}) \mathrm{d} x+\int_{\Omega}(\alpha \Delta S+u \cdot \nabla S)(-D T \cdot \mathbf{V}) \mathrm{d} x \\
& -\int_{\Omega}(-D u \cdot \mathbf{V}) \cdot \nabla T S \mathrm{~d} x-\int_{\Gamma_{s}}(\alpha \nabla T \cdot \nabla S+u \cdot \nabla T S) \mathbf{V}_{n} \mathrm{~d} s-\int_{\Gamma_{s}} u \cdot(-D T \cdot \mathbf{V}) S \cdot n \mathrm{~d} s-\int_{\Gamma_{s}} \alpha \nabla T \cdot(-D S \cdot \mathbf{V}) \cdot n \mathrm{~d} s \\
& -\int_{\Gamma_{s}} \alpha \nabla S \cdot(-D T \cdot \mathbf{V}) \cdot n \mathrm{~d} s \\
= & \int_{\Omega}(\Delta T-\lambda j \cdot v)(-D T \cdot \mathbf{V}) \mathrm{d} x-\int_{\Omega} \nabla T S \cdot(-D u \cdot \mathbf{V}) \mathrm{d} x \\
& +\int_{\Gamma_{s}}(\alpha \nabla T \cdot \nabla S+u \cdot \nabla T S) \mathbf{V}_{n} \mathrm{~d} s-\int_{\Gamma_{s}} u \cdot(-D T \cdot \mathbf{V}) S \cdot n \mathrm{~d} s \\
& -\int_{\Gamma_{s}} \alpha \nabla T \cdot(-D S \cdot \mathbf{V}) \cdot n \mathrm{~d} s-\int_{\Gamma_{s}} \alpha \nabla S \cdot(-D T \cdot \mathbf{V}) \cdot n \mathrm{~d} s .
\end{aligned}
$$

Finally, we have the boundary expression for the Eulerian derivative of $J(\Omega)$ :

$$
\mathrm{d} J(\Omega ; V)=2 v \int_{\Gamma_{s}}\left[\epsilon(u): \in(v)-|\epsilon(u)|^{2}\right] \mathbf{V}_{n} \mathrm{~d} s+\frac{1}{2} \int_{\Gamma_{s}}|\nabla T|^{2} \mathbf{V}_{n} \mathrm{~d} s+\int_{\Gamma_{s}} \alpha \nabla T \cdot \nabla S \mathbf{V}_{n} \mathrm{~d} s
$$

According to (32), we derive the expression of the shape gradient for the cost functional:

$$
\nabla J=\left[2 \nu\left(\epsilon(u): \in(v)-|\epsilon(u)|^{2}\right)+\frac{1}{2}|\nabla T|^{2}+\alpha \nabla T \cdot \nabla S\right] n .
$$

\section{Numerical Examples}

This section is devoted to present the numerical algorithm and examples for the shape optimization problem in two dimensions.
We consider the optimal design of a body immersed in a Stokes-Brinkman flow and aim at reducing the dissipation energy acting on its surface. Namely, we solve the minimization problem

$$
\min _{\Omega \in \mathcal{O}} J(\Omega)=2 v \int_{\Omega}|\epsilon(u)|^{2} \mathrm{~d} x+\frac{1}{2} \int_{\Omega}|\nabla T|^{2} \mathrm{~d} x,
$$

subject to (6)-(13).

For the minimization problem (50), we rather work with the following minimization problem:

$$
\min _{\Omega \in \mathbb{R}^{2}} G(\Omega)=J(\Omega)+l A(\Omega),
$$


Choose an initial shape $\Omega_{0}$ and initial step $h_{0}$ and a Lagrangian multiplier $L_{0}$ while $\epsilon_{\mathrm{re}} \leq \epsilon$, do

Step 1: solve state system (6)-(13).

Step 2: compute adjoint system (27) and (28).

Step 3: evaluate the cost functional.

Step 4: compute the descent direction $d_{k}$ by (56).

Step 5: set $\Omega_{k+1}=\left(\mathrm{I}+h_{k} d_{k}\right) \Omega_{k}$ and a suitable Lagrange multiplier $l_{k+1}$, where $h_{k}$ is a small positive real number. en d while

Algorithm 1: Iterative algorithm for shape optimal control.

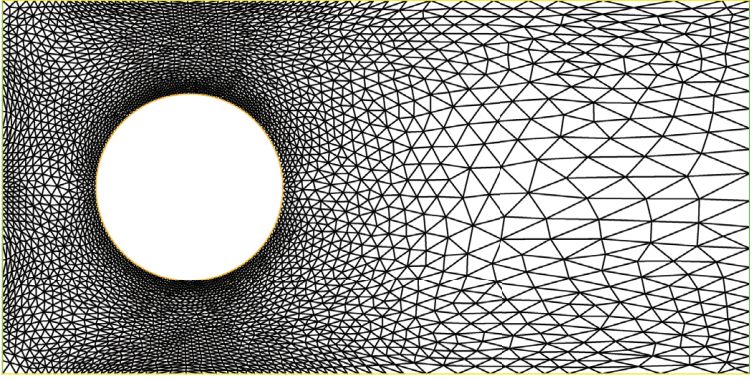

(a)

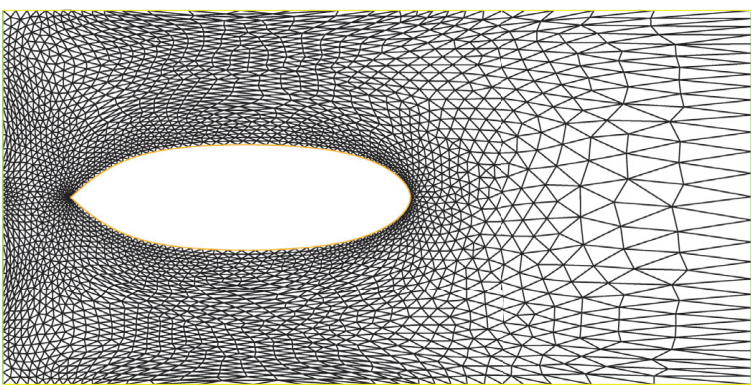

(c)

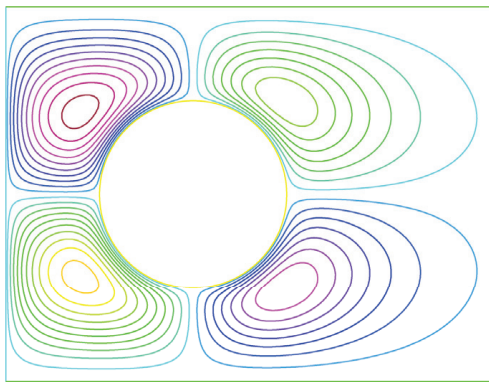

(e)

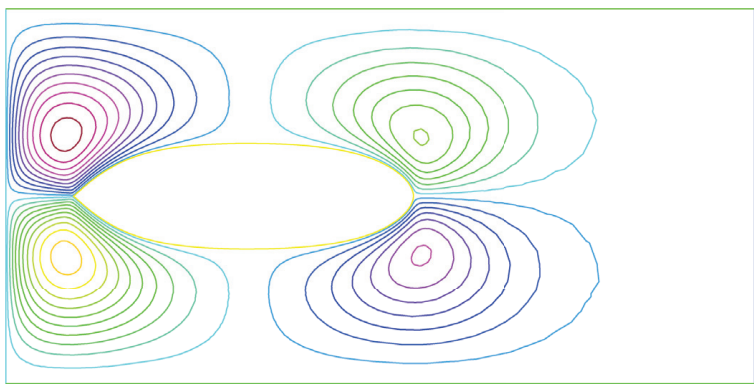

(g)

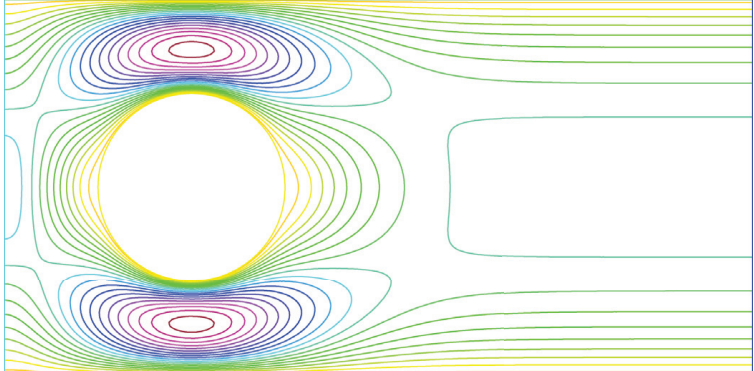

(b)

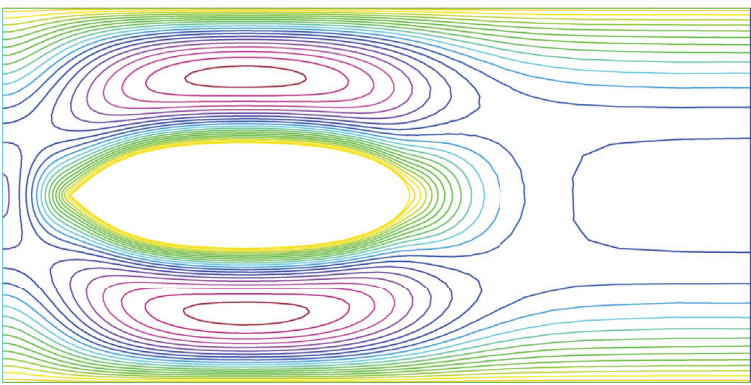

(d)

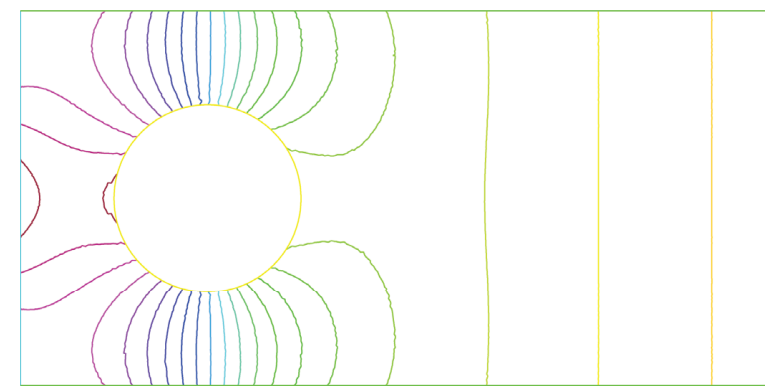

(f)

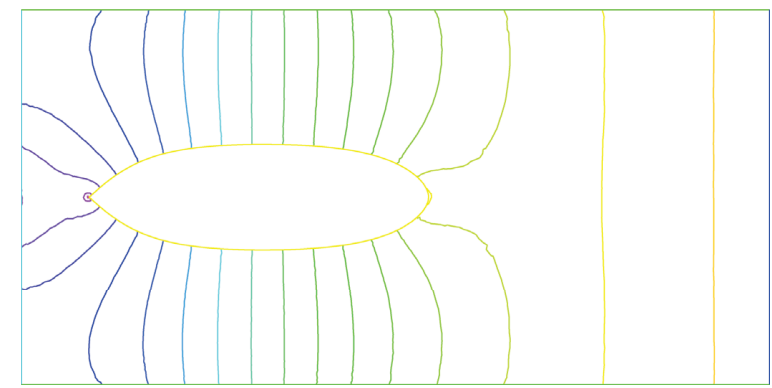

(h)

FIgURE 1: Case 1: comparison of the initial shape and optimal shape (Reynolds number $=1000)$. (a) Mesh for initial shape. (b) $u_{1}$ for initial shape. (c) Mesh for optimal shape. (d) $u_{1}$ for optimal shape. (e) $u_{2}$ for initial shape. (f) $p$ for initial shape. (g) $u_{2}$ for optimal shape. (h) $p$ for optimal shape. 


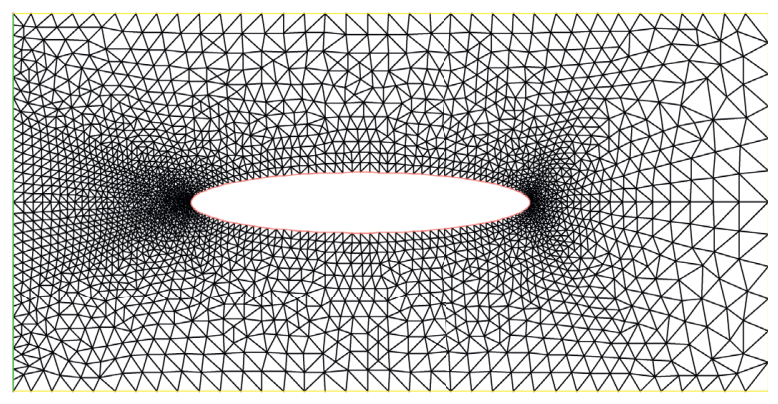

(a)

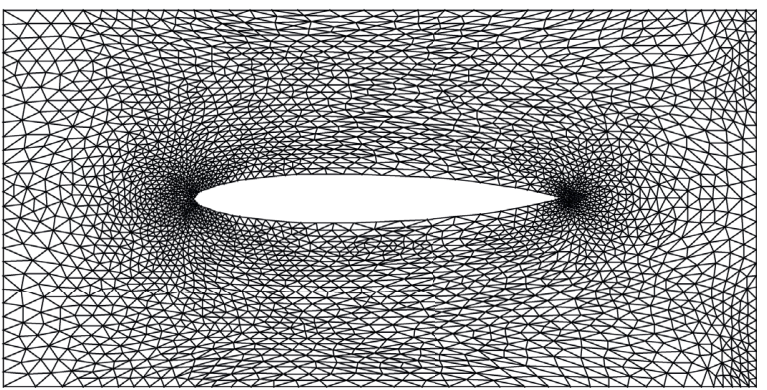

(c)

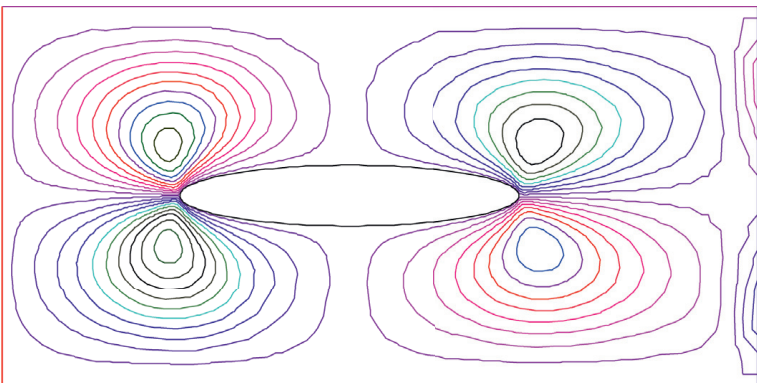

(e)

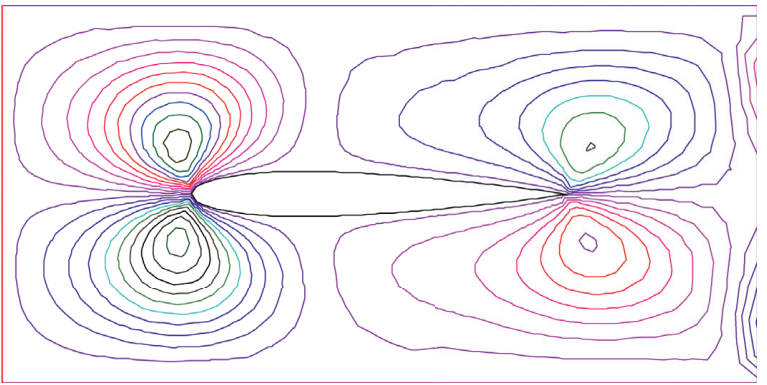

(g)

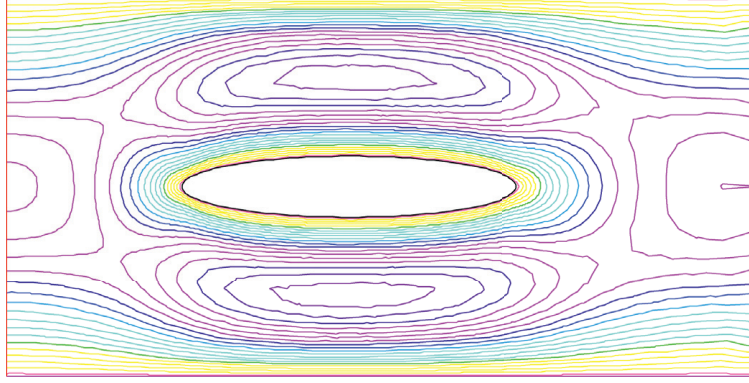

(b)

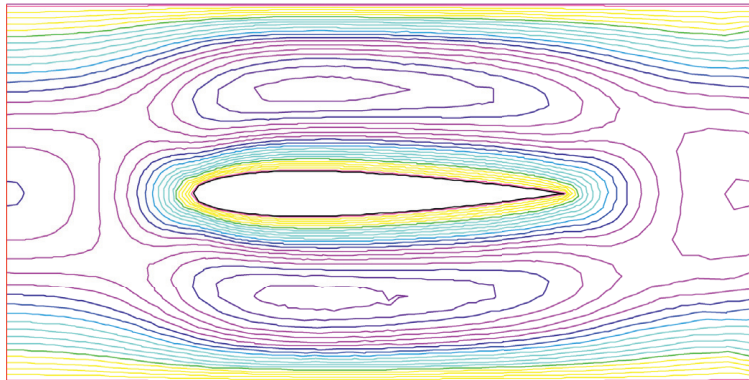

(d)

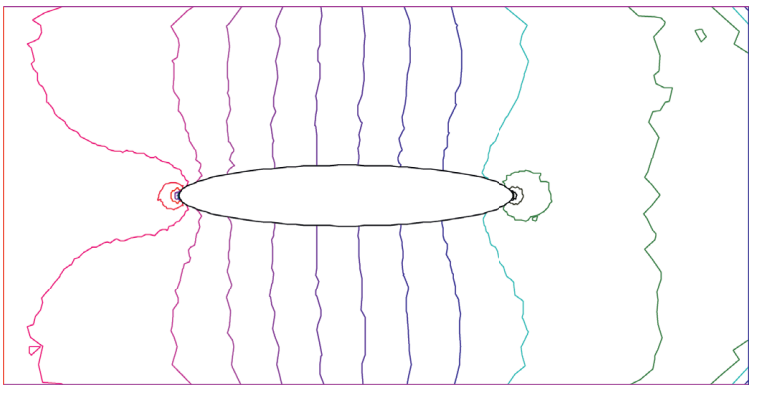

(f)

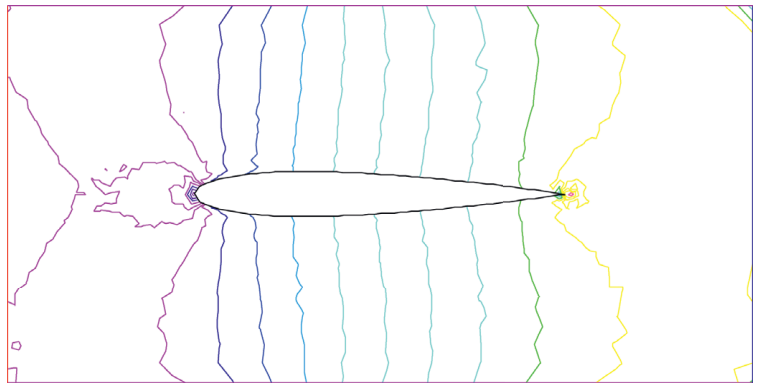

(h)

Figure 2: Case 2: comparison of the initial shape and optimal shape (Reynolds number $=500$ ). (a) Mesh for initial shape. (b) $u_{1}$ for initial shape. (c) Mesh for optimal shape. (d) $u_{1}$ for optimal shape. (e) $u_{2}$ for initial shape. (f) $p$ for initial shape. (g) $u_{2}$ for optimal shape. (h) $p$ for optimal shape.

where $A(\Omega):=\int_{\Omega} \mathrm{d} x \mathrm{~d} y, l$ is a positive Lagrangian multiplier, and $G(\Omega)$ satisfies the following equation:

$$
\mathrm{d} G=\int_{\Gamma_{s}} \nabla G \cdot \mathbf{V} \mathrm{d} s,
$$

where $\mathrm{d} G$ is the shape gradient with

$$
\mathrm{d} G=\left[2 v\left(\epsilon(u): \in(v)-|\epsilon(u)|^{2}\right)+\frac{1}{2}|\nabla T|^{2}+\alpha \nabla T \cdot \nabla S+l\right] n .
$$

Taking no account of regularization, a descent direction is sought by 


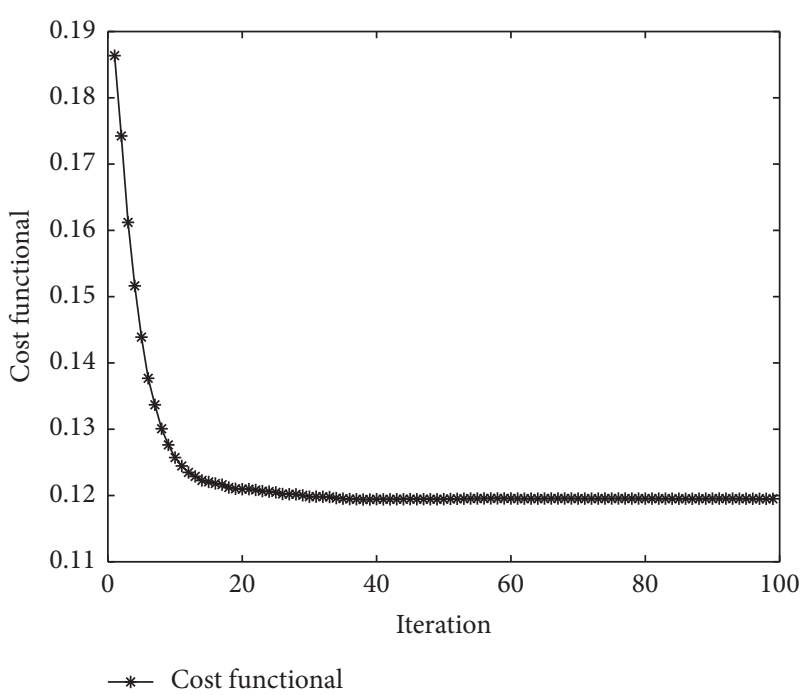

FIgURE 3: Case 1: convergence history of the cost functional $($ Reynolds number $=1000)$.

$$
\mathbf{V}=-h_{k} \nabla G
$$

and then the shape of domain $\Omega$ can be updated as

$$
\Omega_{k}=\left(I+h_{k} \mathbf{V}\right) \Omega,
$$

where $h_{k}$ is a small descent step at $k$-th iteration. We obtain the iterative scheme:

$$
J_{k+1}=J_{k}-h_{k}\left(\nabla J_{k}, \nabla J_{k}\right)_{0}, \quad J_{k}:=J\left(\Omega_{k}\right) .
$$

To avoid shape oscillations, we have to project or smooth the variation into $H^{1}(\Omega)$. Therefore, we choose the descent direction $d \in H^{1}(\Omega)^{2}$ which is the unique solution of the problem

$$
\int_{\Omega} D d: D \mathbf{V d} x=-\mathrm{d} J(\Omega ; \mathbf{V}), \quad \forall \mathbf{V} \in H^{1}(\Omega)^{2} .
$$

It is obvious that $d$ is a descent direction which guarantees the decrease of the cost functional $J(\Omega)$. The computation of $d$ is seemed as a regularization of the shape gradient.

Then, we consider how to choose the Lagrangian multiplier $l$ in the optimization problem. In order to satisfy the fixed constraint, the value of $l$ is updated at each iteration. As a result of the high cost in moving the mesh, we do not impose exactly the volume constraint before convergence. If the present area is smaller than the target area, we decrease the multiplier $l$; otherwise, we increase it. We suppose

$$
\mathrm{d} G(\Omega ; \mathbf{V})=\mathrm{d} J(\Omega ; \mathbf{V})+l \mathrm{~d} V(\Omega ; \mathbf{V})=0,
$$

at least in the average sense on the boundary $\Gamma_{s}$,

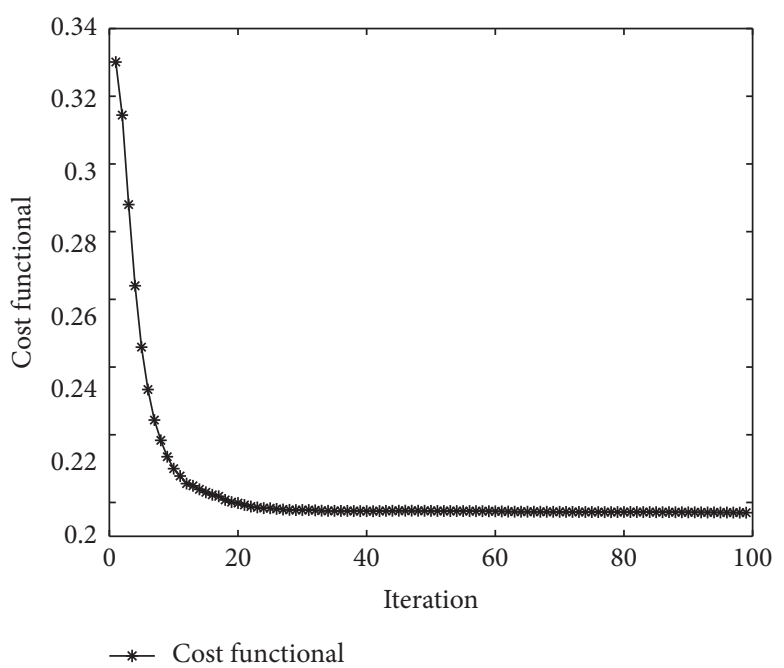

Figure 4: Case 2: convergence history of the cost functional (Reynolds number $=500$ ).

$$
l=-\frac{\int_{\Gamma_{s}} \mathrm{~d} J \mathrm{~d} s}{\int_{\Gamma_{s}} \mathrm{~d} s} .
$$

Therefore, we update the Lagrange multiplier by

$$
l_{k+1}=\frac{\left(l_{k+l}+l\right)}{2}+m \frac{\left|A\left(\Omega_{k}\right)-A_{s}(\Omega)\right|}{A_{s}(\Omega)},
$$

where $m$ is a small positive parameter and $A_{s}(\Omega)$ denotes the target area.

We propose the numerical algorithm for solving the shape optimal problem in a Stokes-Brinkman flow with convective transfer. The algorithm is terminated when the relative decrease (denoted by $\epsilon_{\mathrm{re}}$ ) of two consecutive objective is less than a given tolerance $\epsilon$ (Algorithm 1).

We restrict the shape optimal problem posed on a bounded rectangular domain by introducing an artificial boundary $\partial D$, and $\Omega=D \backslash \bar{S}$ is the effective domain with its boundary $\partial \Omega=\Gamma_{n} \cup \Gamma_{w} \cup \Gamma_{o}$. The fluid enters horizontally from the left boundary $\Gamma_{n}$ and exits from the right boundary $\Gamma_{o}$. We choose the initial shapes of the obstacle $S$ to be different curves:

Case 1: a circle whose center is at origin with radius 0.5 .

Case 2: an elliptic curve: $x=0.45 *$ ost $+0.4, y=0.08 *$ $\sin t, t \in[0,2 \pi]$.

For the two examples, the inflow velocities are assumed to be parabolic with the profiles $g(0, y)=\left(1-y^{4}, 0\right)^{T}$ and $g(0, y)=\left(0.25-y^{2}, 0\right)^{T}$, respectively. Also, the no-slip boundary conditions are imposed at all the other boundaries. The admissible set is defined by

$$
\mathcal{O}=\left\{\Omega \subset \mathbb{R}^{2}: \Gamma_{n} \cup \Gamma_{w} \cup \Gamma_{o} \text { is fixed, the area } A_{\text {target }}(\Omega)=\text { constant }\right\} \text {. }
$$


Figures 1 and 2 show the comparison between the initial shape and optimal shape for the computing meshes, the contours of the velocity $\vec{u}=\left(u_{1}, u_{2}\right)^{T}$, and the pressure $p$ with different Reynolds numbers. Figures 3 and 4 demonstrate that the proposed method is effective, stable, and rapidly convergent. We also observe that when the Reynolds number increases, the cost of the optimization procedure rises due to the increase of computation of the state and adjoint system.

\section{Conclusion}

This work focuses on the optimal shape determination in an incompressible viscous Stokes-Brinkman flow, with the consideration of heat transfer. Based on the adjoint method and the function space parametrization technique, we derive the shape gradient of the cost functional by involving a Lagrangian functional, which plays the key role of design variables in the optimal design framework. Moreover, we propose a gradient-type algorithm for the minimization dissipation energy problem. Finally, we present numerical examples to demonstrate the proposed algorithm is feasible and effective for the quite high Reynolds number problems.

\section{Data Availability}

The data and code used in this study cannot be shared at this time as the data also form a part of an ongoing study.

\section{Conflicts of Interest}

The authors declare that they have no conflicts of interest.

\section{Acknowledgments}

This study was supported by the National Natural Science Foundation of China (grant nos. 11971377, 91730306, and 11701451), Natural Science Foundation of Shaanxi Province (grant nos. 2019JM-367, 2018JQ1077, and 2019JQ-173), and Scientific Research Program funded by Shaanxi Provincial Education Department (grant no. 17JK0787).

\section{References}

[1] S. Milewski, "Determination of the truss static state by means of the combined FE/GA approach, on the basis of strain and displacement measurements," Inverse Problems in Science and Engineering, vol. 27, no. 11, pp. 1537-1558, 2019.

[2] C. O. E. Burg and J. C. Newma III, "Computationally efficient, numerically exact design space derivatives via the complex Taylor's series expansion method," Computers \& Fluids, vol. 32, no. 3, pp. 373-383, 2003.

[3] L. Sherman, A. Taylor, L. Green et al., "First and second order aerodynamic sensitivity derivatives via automatic differentiation with incremental iterative methods," Journal of Computational Physics, vol. 129, no. 2, pp. 307-331, 1994.

[4] S. B. Hazra and A. Jameson, "One-shot pseudo-time method for aerodynamic shape optimization using the Navier-Stokes equations," International Journal for Numerical Methods in Fluids, vol. 68, no. 5, pp. 564-581, 2012.
[5] L. Kusch, T. Albring, A. Walther, and N. R. Gauger, "A oneshot optimization framework with additional equality constraints applied to multi-objective aerodynamic shape optimization," Optimization Methods and Software, vol. 33, no. 4-6, pp. 694-707, 2018.

[6] P. Fulmanski, A. Laurain, J. Scheid et al., "Level set method with topological derivatives in shape optimization," International Journal of Computer Mathematics, vol. 85, no. 10, pp. 1491-1514, 2018.

[7] S. Zhu, Q. Wu, and C. Liu, "Shape and topology optimization for elliptic boundary value problems using a piecewise constant level set method," Applied Numerical Mathematics, vol. 61, no. 6, pp. 752-767, 2011.

[8] W. Yan, Y. He, and Y. Ma, "A numerical method for the viscous incompressible Oseen flow in shape reconstruction," Applied Mathematical Modelling, vol. 36, no. 1, pp. 301-309, 2012.

[9] B. Mohammadi and O. Pironneau, "Shape optimization in fluid mechanics," Annual Review of Fluid Mechanics, vol. 36, no. 1, pp. 255-279, 2004.

[10] M. Giles and N. Pierce, "An introduction to the adjoint approach to design," Flow Turbulence and Combustion, vol. 65, no. 3-4, pp. 393-415, 2000.

[11] E. Katamine, H. Azegami, T. Tsubata, and S. Itoh, "Solution to shape optimization problems of viscous flow fields," International Journal of Computational Fluid Dynamics, vol. 19, no. 1, pp. 45-51, 2005.

[12] A. Jameson, "Computational algorithms for aerodynamic analysis and design," Applied Numerical Mathematics, vol. 13, no. 5, pp. 383-422, 1993.

[13] D. N. Srinath and S. Mittal, "An adjoint method for shape optimization in unsteady viscous flows," Journal of Computational Physics, vol. 229, no. 6, pp. 1994-2008, 2010.

[14] H. Yagi and M. Kawahara, "Optimal shape determination of a body located in incompressible viscous fluid flow," Computer Methods in Applied Mechanics and Engineering, vol. 196, no. 49-52, pp. 5084-5091, 2007.

[15] Y. Ogawa and M. Kawahara, "Shape optimization of body located in incompressible viscous flow based on optimal control theory," International Journal of Computational Fluid Dynamics, vol. 17, no. 4, pp. 243-251, 2003.

[16] H. Heck, G. Uhlmann, G. Uhlmann, and J.-N. Wang, "Reconstruction of obstacles immersed in an incompressible fluid," Inverse Problems \& Imaging, vol. 1, no. 1, pp. 63-76, 2007.

[17] D. Chenais, J. Monnier, and J. P. Vila, "Shape optimal design problem with convective and radiative heat transfer: analysis and implementation," Journal of Optimization Theory and Applications, vol. 110, no. 1, pp. 75-117, 2001.

[18] W. Yan, Y. He, and Y. Ma, "Shape inverse problem for the twodimensional unsteady stokes flow," Numerical Methods for Partial Differential Equations, vol. 26, no. 3, pp. 690-701, 2010.

[19] F. Caubet and M. Dambrine, "Localization of small obstacles in Stokes flow," Inverse Problems, vol. 28, no. 10, p. 105007, 2012.

[20] A. Abda, M. Hassine, M. Jaoua et al., "Topological sensitivity analysis for the location of small cavities in stokes flow," SIAM Journal on Control and Optimization, vol. 48, no. 5, pp. 2871-2900, 2009.

[21] J. Lions, Optimal Control of Systems Governed by Partial Differential Equations, Springer-Verlag, New-York, NY, USA, 1971.

[22] O. Pironneau, Optimal Shape Design by Local Boundary Variations, Springer-Verlag, Berlin, Germany, 1988.

[23] M. C. Delfour and J.-P. Zolésio, Shapes and Geometries: Analysis, Differential Calculus, and Optimization, Advance in Design and Control, Society for Industrial and Applied Mathematics, Philadelphia, PA, USA, 2001. 